\title{
NEVANLINNA, SIEGEL, AND CREMER
}

\author{
YÛSUKE OKUYAMA
}

\begin{abstract}
We study an irrationally indifferent cycle of points or circles of a rational function, which is either Siegel or Cremer by definition. We invent a new argument from the viewpoint of the Nevanlinna theory. Using this argument, we give a clear interpretation of some Diophantine quantity associated with an irrationally indifferent cycle. This quantity turns out to be Nevanlinnatheoretical. As a consequence, we show that an irrationally indifferent cycle is Cremer if this Nevanlinna-theoretical quantity does not vanish.
\end{abstract}

\section{INTRODUCTION}

Let $f$ be a rational function of degree $\geq 2$ and $f^{k}:=f^{\circ k}$ for $k \in \mathbb{N}$. The Fatou set $F(f)$ is defined by the set of all points of $\hat{\mathbb{C}}$ at which $\left\{f^{n}\right\}_{n \in \mathbb{N}}$ is normal in the Montel sense, and the Julia set $J(f)$ is defined by the complement of $F(f)$ in $\hat{\mathbb{C}}$. Both $F(f)$ and $J(f)$ are completely invariant, that is, their image and preimage by $f$ equal themselves. $F(f)$ is open by definition, so $J(f)$ is closed. Furthermore $J(f)$ is non-empty and perfect.

We call a connected component of $F(f)$ a Fatou component. Every Fatou component is mapped to a Fatou one properly by $f$. A Fatou component $D$ is cyclic if for some $n \in \mathbb{N}, f^{n}(D)=D$. Then the least such $n$ is called the period, and $g:=g_{D}:=f^{n} \mid D$ is the first return map on $D$. On the other hand, a Fatou component is preperiodic if it is not cyclic but for some $n \in \mathbb{N}, f^{n}(D)$ is cyclic.

The classification of cyclic Fatou components is known: $(g, D)$ is an attractive basin if $\left\{g^{n}\right\}_{n=1}^{\infty}$ converges to a point in $D$ locally uniformly on $D$. The parabolic basin is similar, but $\left\{g^{n}\right\}_{n=1}^{\infty}$ converges to a point in the boundary of $D$ locally uniformly on $D$. When $g$ is a proper selfmap of $D$ of degree $\geq 2,(g, D)$ is one of them. When $g$ is a univalent selfmap of $D,(g, D)$ is called a singular domain since people before doubted whether this case actually occurred. In this case, $(g, D)$ is conformally conjugate to an irrational rotation on either a disk or an annulus, and called a Siegel disk or an Herman ring respectively. Hence the singular domain is also called the rotation one. For the details, see [11], [2], [4], [10].

Our main interest is an irrationally indifferent cycle of points or circles.

Definition 1.1 (irrationally indifferent cycle of points or circles). A point $z_{0}$ in $\hat{\mathbb{C}}$ is periodic if for some $p \in \mathbb{N}, f^{p}\left(z_{0}\right)=z_{0}$. The least such $p$ is the period of $z_{0}$, $\left\{f^{n}\left(z_{0}\right)\right\}_{n=1}^{p}$ is a cycle of points, and $\lambda:=\left(f^{p}\right)^{\prime}\left(z_{0}\right)$ is the multiplier of it. This cycle of points is irrationally indifferent if $\lambda=e^{2 \pi i \alpha}$ for some $\alpha \in \mathbb{R}-\mathbb{Q}$.

Date: March 17, 2003.

1991 Mathematics Subject Classification. Primary 30D05; Secondary 30D35, 37F50, 39 B12.

Key words and phrases. Nevanlinna Theory, irrationally indifferent cycle, Siegel cycle, Cremer cycle.

Partially supported by the Sumitomo Foundation. 
A topological circle $S \subset \hat{\mathbb{C}}$ is periodic if for some $p \in \mathbb{N}, f^{p}(S)=S$ and $f^{p} \mid S$ : $S \rightarrow S$ is an orientation-preserving homeomorphism. The least such $p$ is the period of $S,\left\{f^{n}(S)\right\}_{n=1}^{p}$ is a cycle of circles, and $\lambda:=e^{2 \pi i \alpha}$ is the multiplier of it, where $\alpha \in \mathbb{R} / \mathbb{Z}$ is the rotation number (cf. [6]) of a $S^{1}$-homeomorphism $\phi$ which is topologically conjugate to $f^{p} \mid S$. This cycle of circles is irrationally indifferent if $\alpha$ is irrational.

It is known that if irrationally indifferent cycles of points or circles intersect $F(f)$, then they are contained in some rotation domains, which are Fatou components.

Definition 1.2 (Siegel and Cremer cycles). An irrationally indifferent cycle of points or circles is a Siegel cycle if it is contained in $F(f)$. Otherwise it is a Cremer cycle.

We study an unsolved problem with a long history: Given an irrationally indifferent cycle of points or circles, how can we judge whether it is contained in the Fatou set or not?

The following answers the problem in one direction.

Theorem 1.1 (Siegel[17] (1942), Rüssmann, Brjuno[3] (1972), Yoccoz[20] (1996)). Let $\lambda=e^{2 \pi i \alpha}(\alpha \in \mathbb{R}-\mathbb{Q}), f(z)=\lambda z+\cdots$ be an analytic germ at the origin, and $\left\{p_{n} / q_{n}\right\}_{n=0}^{\infty}$ be the sequence of irreducible approximating fractions of $\alpha$ derived from its continued fraction expansion.

If $\alpha$ satisfies one of the following Diophantine conditions:

$$
\sup _{n \geq 0} \frac{\log q_{n+1}}{\log q_{n}}<\infty
$$

a weaker one:

$$
\sum_{n \geq 0} \frac{\log q_{n+1} \log \log q_{n+1}}{q_{n}}<\infty
$$

and the weakest one:

$$
\sum_{n \geq 0} \frac{\log q_{n+1}}{q_{n}}<\infty
$$

then $f$ is analytically linearizable at the origin, that is, the Schröder equation:

$$
h \circ f=R_{\lambda} \circ h,
$$

where $R_{\lambda}(z)=\lambda z$ is the linear term of $f$, holds for some analytic local coordinate $h(z)=z+\cdots$ around the origin.

Corollary 1.1. An irrationally indifferent cycle of points of a rational function is Siegel if its multiplier satisfies the condition $(\mathrm{Br})$.

In the reverse direction,

Theorem 1.2 (Yoccoz[20] (1996), Okuyama[12] (2001)). Let $P$ be a quadratic polynomial. An irrationally indifferent cycles of points (of arbitrary period) of $P$ is Cremer if its multiplier does not satisfy $(\mathrm{Br})$.

Only for quadratic polynomials, the complete answer of the problem is known. The classical Cremer Theorem is a partial answer in the reverse direction. 
Theorem 1.3 (Cremer[5] (1932)). Let $f$ be a rational function of degree $d \geq 2$, and $\mathcal{O}$ be an irrationally indifferent cycle of points of period $p$ and of multiplier $\lambda$.

$\mathcal{O}$ is Cremer if $\lambda$ satisfies

$$
\limsup _{n \rightarrow \infty} \frac{1}{d^{p n}} \log \frac{1}{\left|\lambda^{n}-1\right|}=\infty
$$

It naturally arises:

Fundamental Question. How can we notice such complicate Diophantine conditions as $(\mathrm{Si}),(\mathrm{Rü}),(\mathrm{Br})$ and $(\mathrm{Cr})$ ? What on earth are they?

We shall answer this Fundamental Question for the condition $(\mathrm{Cr})$ and $(\mathrm{Ok})$ below. The left hand side of them turns out to be Nevanlinna-theoretical. From this, it immediately follows that irrationally indifferent cycles of points or circles are Cremer if this Nevanlinna-theoretical quantity does not vanish:

Main Theorem 1 (Criterion for Cremer). Let $f$ be a rational function of degree $d \geq 2$, and $\mathcal{O}$ be an irrationally indifferent cycle of points or circles of period $p$ and of multiplier $\lambda$. If

$$
\limsup _{n \rightarrow \infty} \frac{1}{d^{p n}} \log \frac{1}{\left|\lambda^{n}-1\right|}>0
$$

then $\mathcal{O}$ is Cremer.

In Section 2, we shall study the Nevanlinna theory. We define the pointwise proximity function, the mean proximity and the Valiron exceptionality, and prove the Fundamental Equality for the Valiron exceptionality. This Fundamental Equality shows that the dynamics of a rational function is homogeneous on the whole Riemann sphere. In Section 3, by the Fundamental Equality, we shall prove the Vanishing Theorem, which states that the Valiron exceptionality vanishes for a rational function with non-empty Fatou set. In Section 4, we shall obtain the Natural Equality: the left hand side of (Ok) exactly equals the Valiron exceptionality. Main Theorem 1 is straightforward from both the Vanishing Theorem and the Natural Equality.

First of all, our method of studying this problem answers the Fundamental Question, and then naturally establishes a criterion for Cremer. Hence we find that our method is natural.

ACKNOWLEDGEMENT. The author would like to express his gratitude to Professors Masahiko Taniguchi and Toshiyuki Sugawa for many valuable discussions and advices. He also thanks to Professors Mitsuhiro Shishikura, Kevin M. Pilgrim and Erik Bedford for very useful comments.

\section{NeVAnLinna theORY}

Let $[p, q]$ be the chordal distance between $p, q \in \hat{\mathbb{C}}$ such that $[0, \infty]=1$. For rational functions $f$ and $g$, we define the pointwise proximity function:

$$
w(g, f):=\log \frac{1}{[g, f]},
$$

and the mean proximity:

$$
m(g, f):=\int_{\hat{\mathbb{C}}} w(g, f) d \sigma,
$$

where $\sigma$ is the spherical area measure on $\hat{\mathbb{C}}$ such that $\sigma(\hat{\mathbb{C}})=1$. 
Definition 2.1 ([18], cf. [8]). Let $\mathcal{F}=\left\{f_{k}\right\}_{k=1}^{\infty}$ be a sequence of rational functions such that $d_{k}:=\operatorname{deg} f_{k} \uparrow \infty$ as $k \rightarrow \infty$. For a rational function $g$, we define the Valiron exceptionality:

$$
\operatorname{VE}(g ; \mathcal{F}):=\limsup _{k \rightarrow \infty} \frac{m\left(g, f_{k}\right)}{d_{k}} .
$$

$g$ is Valiron exceptional for $\mathcal{F}$ if $\operatorname{VE}(g ; \mathcal{F})>0$.

Example 1. In the case that $\mathcal{F}=\left\{z^{k}\right\}$ and $g \equiv 0$, it holds that $\operatorname{VE}(g ; \mathcal{F})>0$. Hence $g$ is Valiron exceptional for $\mathcal{F}$.

Main Theorem 2 (Fundamental Equality). Let $f$ be a rational function of degree $d \geq 2$. Then for every positive continuous function $\phi \not \equiv 0$ on $\hat{\mathbb{C}}$,

$$
\operatorname{VE}\left(\operatorname{Id}_{\widehat{\mathbb{C}}} ;\left\{f^{k}\right\}\right)=\limsup _{k \rightarrow \infty} \frac{\int_{\hat{\mathbb{C}}} \phi \cdot w\left(\operatorname{Id}_{\widehat{\mathbb{C}}}, f^{k}\right) d \sigma}{d^{k} \cdot \int_{\hat{\mathbb{C}}} \phi d \sigma} .
$$

Proof. Let $\mathbb{M}(\hat{\mathbb{C}})$ be the set of all Radon measures on $\hat{\mathbb{C}}$. For rational functions $f$ and $g$, we define the root measure

$$
(f-g)^{*} \delta_{0}:=\sum_{f \zeta=g \zeta} \delta_{\zeta} \in \mathbb{M}(\hat{\mathbb{C}}),
$$

taking into account the multiplicities of roots, where $\delta_{\zeta} \in \mathbb{M}(\hat{\mathbb{C}})$ is the Dirac measure at $\zeta \in \hat{\mathbb{C}}$. For a rational function $f$ and $\mu \in \mathbb{M}(\hat{\mathbb{C}})$, the pullback measure $f^{*} \mu \in \mathbb{M}(\hat{\mathbb{C}})$ is defined as

$$
f^{*} \mu(U)=\int_{\widehat{\mathbb{C}}}\left((f-z)^{*} \delta_{0}\right)(U) d \mu(z),
$$

where $U$ is a Borel set in $\hat{\mathbb{C}}$. For $\mu \in \mathbb{M}(\hat{\mathbb{C}})$, we consider the chordal logarithmic potential

$$
P_{\mu}(z):=\int_{\hat{\mathbb{C}}} \log \frac{1}{[w, z]} d \mu(w)
$$

on $\hat{\mathbb{C}}$.

Lemma 2.1 (Riesz decomposition). For rational functions $f$ and $g$,

$$
w(f, g)=P_{(f-g) * \delta_{0}}-P_{f^{*} \sigma}-P_{g^{*} \sigma}+m(f, g) .
$$

Proof. By a Möbius conjugation, we assume $f(\infty) \neq g(\infty), f^{\prime}(\infty) \neq 0$, and $g^{\prime}(\infty) \neq 0$ without loss of generality. Since $w(f, g)$ is $\delta$-subharmonic on $\mathbb{C}$, it has the Riesz decomposition (cf. [13]):

$$
w(f, g)=P_{(f-g)^{*} \delta_{0}}-P_{f^{*} \sigma}-P_{g^{*} \sigma}+c_{f, g},
$$

where $c_{f, g}$ is harmonic on $\mathbb{C}$. Since $c_{f, g}=O(1)$ as $z \rightarrow \infty, c_{f, g}$ is a constant by the Liouville theorem. Since the $\sigma$-mean of $P_{(f-g)^{*} \delta_{0}}-P_{f^{*} \sigma}-P_{g^{*} \sigma}$ on $\hat{\mathbb{C}}$ vanishes, it follows that $c_{f, g}=m(f, g)$.

Let $\phi$ be a continuous function on $\hat{\mathbb{C}}$. By Lemma 2.1, we have

$$
\begin{aligned}
& \frac{1}{d^{k}} \int_{\hat{\mathbb{C}}} \phi \cdot w\left(\operatorname{Id}_{\hat{\mathbb{C}}}, f^{k}\right) d \sigma \\
= & \frac{1}{d^{k}} \int_{\hat{\mathbb{C}}} \phi \cdot\left(P_{\left(f^{k}-\mathrm{Id}_{\hat{\mathbb{C}}}\right)^{*} \delta_{0}}-P_{\left(f^{k}\right)^{*} \sigma}-P_{\sigma}\right) d \sigma+\frac{m\left(\operatorname{Id}_{\hat{\mathbb{C}}}, f^{k}\right)}{d^{k}} \int_{\hat{\mathbb{C}}} \phi d \sigma,
\end{aligned}
$$


and by the Fubini theorem, the first term of $(*)$ equals

$$
\int_{\hat{\mathbb{C}}}\left(\int_{\hat{\mathbb{C}}} \phi(z) \log \frac{1}{[w, z]} d \sigma(z)\right) d \frac{\left(f^{k}-\mathrm{Id}_{\hat{\mathbb{C}}}\right)^{*} \delta_{0}-\left(f^{k}\right)^{*} \sigma-\sigma}{d^{k}}(w) .
$$

Theorem 2.1 ([7] and [9]). Both $\left(f^{k}-\mathrm{Id}_{\hat{\mathbb{C}}}\right)^{*} \delta_{0} / d^{k}$ and $\left(f^{k}\right)^{*} \sigma / d^{k}$ converge to the same element of $\mathbb{M}(\hat{\mathbb{C}})$ as $k \rightarrow \infty$ weakly.

Remark 2.1. The limit measure $\mu_{f}$ in Theorem 2.1 is said to be balanced since $f^{*} \mu_{f} / d=\mu_{f}$. It has many interesting dynamical properties.

From Theorem 2.1, we have that the first term of $\left(^{*}\right)$ converges to 0 as $k \rightarrow \infty$. It completes the proof of Main Theorem 2.

\section{The FAtou And Julia strategy}

We are able to calculate the Valiron exceptionalities through the Fatou and Julia strategy.

Main Theorem 3 (Vanishing Theorem). Let $f$ be a rational function of degree $\geq 2$ such that $F(f) \neq \emptyset$. Then $\operatorname{VE}\left(\operatorname{Id}_{\widehat{\mathbb{C}}} ;\left\{f^{k}\right\}\right)=0$.

Proof. It follows from $\operatorname{deg} f \geq 2$ that there exists a Fatou component either noncyclic or non-singular. Hence there exist a positive continuous function $\phi \not \equiv 0$ and $r \in(0,1)$ such that for every $k \in \mathbb{N}$,

$$
\inf \left\{[z, w] ; z \in \operatorname{supp} \phi, w \in f^{k}(\operatorname{supp} \phi)\right\}>r .
$$

From this, it follows that

$$
\int_{\widehat{\mathbb{C}}} \phi \cdot w\left(\operatorname{Id}_{\hat{\mathbb{C}}}, f^{k}\right) d \sigma \leq \log \frac{1}{r} \cdot \int_{\widehat{\mathbb{C}}} \phi d \sigma
$$

which concludes that $\operatorname{VE}\left(\operatorname{Id}_{\widehat{\mathbb{C}}} ;\left\{f^{k}\right\}\right)=0$ from the Fundamental Equality.

\section{Siegel AND CREmer CyCles}

Let $f$ be a rational function of degree $d \geq 2$. From now on, cycles and Fatou components are always of $f$.

For a rotation domain $D$, there exists, by definition, a conformal map $h$ from $D$ onto either $\mathbb{D}$ or an annulus and $\lambda=e^{2 \pi i \alpha}(\alpha \in \mathbb{R}-\mathbb{Q})$ such that for the first return map $g=g_{D}$,

$$
h \circ g=R_{\lambda} \circ h
$$

on $D$. Here $R_{\lambda}(z)=\lambda z$ as Section 1. $h$ is called a linearizing map of $D$ and $\lambda$ the rotation number.

Notation. $A \asymp B$ means $A / C<B<C A$ for some implicit constant $C$.

Remark 4.1. Our method of studying multipliers of irrationally indifferent cycles of circles dispenses with such quasiconformal surgeries as in [14].

The left hand side of $(\mathrm{Ok})$ in Main Theorem 1 turns out to be the Valiron exceptionality. 
Main Theorem 4 (Natural Equality). Let $\mathcal{O}$ be an irrationally indifferent cycle of points or circles of period $p$ and of multiplier $\lambda$. If $\mathcal{O}$ is Siegel, then

$$
\limsup _{k \rightarrow \infty} \frac{1}{d^{p k}} \log \frac{1}{\left|\lambda^{k}-1\right|}=\operatorname{VE}\left(\operatorname{Id}_{\widehat{\mathbb{C}}} ;\left\{f^{p k}\right\}\right)
$$

Proof. Let $C$ be a connected component of $\mathcal{O}$. Then there exists a rotation domain $D$ such that $D \supset C$. Let $h$ be a linearizing map of $D$ and $g=f^{p} \mid D$ the first return map. Clearly the rotation number of $D$ equals the multiplier $\lambda$ of $\mathcal{O}$.

By a Möbius conjugation, we assume $D \subset \mathbb{C}$ without loss of generality. There exists a positive continuous function $\phi \not \equiv 0$ such that supp $\phi \subset D$ and $h(\operatorname{supp} \phi) \not \supset 0$. Since $\overline{\bigcup_{k \in \mathbb{N}} g^{k}(\operatorname{supp} \phi)}$ is compact in $D$, we have:

$$
\left[g^{k}(z), z\right] \asymp\left|g^{k}(z)-z\right| \asymp\left|h \circ g^{k}(z)-h(z)\right|=\left|\lambda^{k}-1\right| \cdot|h(z)|,
$$

where the implicit constants are independent of $k \in \mathbb{N}$ and $z \in \operatorname{supp} \phi$. Hence

$$
\frac{\int_{\hat{\mathbb{C}}} \phi \cdot w\left(\operatorname{Id}_{\hat{\mathbb{C}}}, f^{p k}\right) d \sigma}{d^{p k} \cdot \int_{\hat{\mathbb{C}}} \phi d \sigma}=\frac{1}{d^{p k}} \log \frac{1}{\left|\lambda^{k}-1\right|}+\frac{\int_{\hat{\mathbb{C}}} \phi \cdot \log \frac{1}{|h|} d \sigma}{d^{p k} \cdot \int_{\hat{\mathbb{C}}} \phi d \sigma}+O\left(d^{-p k}\right)
$$

as $k \rightarrow \infty$. It easily follows from $h(\operatorname{supp} \phi) \not \ngtr 0$ that the second term tends to 0 as $k \rightarrow \infty$. Hence the proof is completed by the Fundamental Equality.

Now the proof of Main Theorem 1 is straightforward:

Proof of Main Theorem 1. If $\mathcal{O}$ is Siegel, then $F(f) \neq \emptyset$. Hence from the Vanishing Theorem,

$$
\operatorname{VE}\left(\operatorname{Id}_{\widehat{\mathbb{C}}} ;\left\{f^{p k}\right\}\right) \leq \operatorname{VE}\left(\operatorname{Id}_{\hat{\mathbb{C}}} ;\left\{f^{k}\right\}\right)=0 .
$$

It contradicts (Ok) by the Natural Equality.

Remark 4.2. The Natural Equality in Main Theorem 4 is the very answer to the Fundamental Question in Section 1 for the condition (Ok). As we have just seen in the above, Main Theorem 1 is straightforward from this Natural Equality and the Vanishing Theorem.

In the case of polynomials, Pierre Tortrat showed in [19] a similar result to Main Theorem 1 by using a potential theoretical argument.

We also obtain a priori bounds of the rotation numbers of rotation domains.

Main Theorem 5. The rotation numbers of no rotation domains satisfy (Ok).

Proof. A rotation domain contains Siegel cycles of circles whose multipliers equal its rotation number. Hence they do not satisfy (Ok) by Main Theorem 1.

Remark 4.3. When the rotation domain is an Herman ring, by quasiconformal surgery of it (cf. [14], [15] and [16]), we obtain a rational function $\tilde{f}$ whose degree is less than that of $f$ and which has a Siegel disk with the same rotation number as the original Herman ring of $f$. Hence by applying Main Theorem 5 to $\tilde{f}$ rather than $f$, a stronger conclusion than Main Theorem 5 follows.

Finally, we note that Cremer cycles of circles do not always satisfy (Ok). A normalized cubic critical Blaschke product, e.g.,

$$
f_{\theta}(z):=e^{i \theta} z^{2} \frac{1-\frac{1}{3} z}{z-\frac{1}{3}}
$$

where $\theta$ is chosen such that $S^{1}$ is an irrationally indifferent cycle of circles of period one, is never Siegel since there exists a critical point on $S^{1}$. Other example is: 
Main Theorem 6 (Jordan boundaries of rotation domains). Let $\mathcal{O}$ be an irrationally indifferent cycle of circles which are the boundary of a cycle $\mathcal{C}$ of rotation domains. Then the multiplier of $\mathcal{O}$ does not satisfy $(\mathrm{Ok})$.

Proof. Let $D$ be a connected component of $\mathcal{C}$ and $h$ its linearizing map. The Carathéodory theory (or the Ahlfors-Beurling extremal length method [1]) says that $h$ extends to a homeomorphism from $\bar{D}$ to $\overline{h(D)}$. Hence by the equation (1), the multiplier of $\mathcal{O}$ equals the rotation number of $\mathcal{C}$. By Main Theorem 5, it does not satisfy (Ok).

\section{REFERENCES}

[1] Ahlfors, L. V. Conformal invariants: topics in geometric function theory, McGraw-Hill Book Co., New York (1973), McGraw-Hill Series in Higher Mathematics.

[2] Beardon, A. F. Iteration of rational functions, Vol. 132 of Graduate Texts in Mathematics, Springer-Verlag, New York (1991), Complex analytic dynamical systems.

[3] Brjuno, A. D. Analytical form of differential equations, Trans. Moscow Math. Soc., 25 (1971), 199-239.

[4] Carleson, L. and Gamelin, T. W. Complex Dynamics, Springer-Verlag (1993).

[5] Cremer, H. Über die Schrödersche Funktionalgleichung und das Schwarzsche Eckenabbildungsproblem, Math.-phys. Klasse, 84 (1932), 291-324.

[6] Melo, de W. and Strien, van S. One-dimensional dynamics, Vol. 25 of Ergebnisse der Mathematik und ihrer Grenzgebiete (3) [Results in Mathematics and Related Areas (3)], Springer-Verlag, Berlin (1993)

[7] ERËmenko, A. E. and Sodin, M. L. Iterations of rational functions and the distribution of the values of Poincaré functions, Teor. Funktsǐ Funktsional. Anal. i Prilozhen., 53 (1990), $18-25$.

[8] Laine, I. Nevanlinna theory and complex differential equations, Vol. 15 of de Gruyter Studies in Mathematics, Walter de Gruyter \& Co., Berlin (1993).

[9] LJubich, M. J. Entropy properties of rational endomorphisms of the Riemann sphere, Ergodic Theory Dynam. Systems, 3, 3 (1983), 351-385.

[10] Milnor, J. Dynamics in one complex variable, Vieweg (1999).

[11] Morosama, S., Nishimura, Y., Taniguchi, M. and Ueda, T. Holomorphic Dynamics, Cambredge studies in advanced mathematics 66 (1999).

[12] OKUYAмA, Y. Non-linearizability of $n$-subhyperbolic polynomials at irrationally indifferent fixed points, J. Math. Soc. Japan, 53, 4 (2001), 847-874.

[13] Ransford, T. Potential theory in the complex plane, Cambridge University Press, Cambridge (1995).

[14] Shishikura, M. On the quasiconformal surgery of rational functions, Ann. Scient. Éc Norm Sup., 4 $^{e}$ série, 20 (1987), 1-29.

[15] Shishikura, M. Trees associated with the configuration of Herman rings, Ergodic Theory Dynam. Systems, 9, 3 (1989), 543-560.

[16] Shishikura, M. A new tree assotiated with Herman rings, Sūrikaisekikenkyūsho Kōkyūroku, 1269 (2002), 74-92.

[17] Siegel, C. L. Iterations of analytic functions, Ann. of Math., 43 (1942), 807-812.

[18] Sodin, M. Value distribution of sequences of rational functions, Entire and subharmonic functions, Vol. 11 of Adv. Soviet Math., Amer. Math. Soc., Providence, RI (1992), 7-20.

[19] Tortrat, P. Aspects potentialistes de l'itération des polynômes, Séminaire de Théorie du Potentiel, Paris, No. 8, Vol. 1235 of Lecture Notes in Math., Springer, Berlin (1987), 195-209.

[20] Yoccoz, J.-C. Théorèm de Siegel, nombres de Bruno et polynômes quadratiques, Astérisque, 231 (1996), 3-88.

Department of Mathematics, Faculty of Science, Shizuoka University, Shizuoka 4228529 JAPAN

E-mail address: syokuya@ipc.shizuoka.ac.jp 